\title{
A resposta de observação e os estudos sobre discriminação nas décadas de 1930 e 1940
}

Peter Endemann

\section{RESUMO}

A investigação empírica sobre o processo da discriminação realizada nas décadas de 1930 e 1940 foi acompanhada por um longo debate, conhecido como a Controvérsia Continuidade vs. Descontinuidade. Questões sobre a natureza seletiva da discriminação e o papel de respostas sensoriais compuseram o problema de pesquisa de Wyckoff, que culminaria na elaboração do conceito e do delineamento experimental da resposta de observação. Com o objetivo de regatar o contexto histórico de surgimento da resposta de observação, a presente pesquisa foi conduzida com base nas referências dos artigos originais de Wyckoff. 0 delineamento de observação permitiu a obtenção de medidas independentes de duas classes de respostas importantes para o estabelecimento da discriminação. 0 papel das respostas sensoriais e, especificamente, da resposta de observação foram abordados experimentalmente a partir de condições experimentais, nas quais o contato do organismo com os estímulos discriminativos não foi garantido ou favorecido pelo arranjo de estímulo.

Palavras-chave: Discriminação; resposta de orientação; resposta de observação; delineamento experimental; história

\section{ABSTRACT}

\section{Observing response and discrimination learning in 1930s and 1940s}

Empirical research on discrimination conducted in 1930s and 1940s was accompanied by a long debate, known as Continuity vs. Non-continuity Controversy. Questions about the selective nature of discrimination and the role of sensory responses composed the research problem that would culminate in the elaboration of the concept and the experimental design of the observing response. In order to present the historical context of the observing response, the present work was conducted based on the references of Wyckoff's original articles. Observing design allowed independent measurements of two classes of responses. The role of sensory responses and specifically observing response were experimentally investigated from experimental conditions in which the contact of the organism with discriminative stimuli was not guaranteed or favored by the stimulus arrangement.

Keywords: Discrimination; orientation response; observing response; experimental design; history

A investigação empírica sobre o processo da discriminação realizada nas décadas de 1930 e 1940 foi acompanhada por um longo debate, a partir do qual a natureza seletiva do processo e o papel das respostas sensoriais (e.g., respostas de orientação dos órgãos receptores) foram discutidos (Krechevsky, 1932, 1938; Lashley, 1929, 1942; Spence, 1936, 1940, 1945). Esse debate ficou conhecido como a Controvérsia Continuidade vs. Descontinuidade (Continuity and noncontinuity controversy). Dessa forma, o problema de pesquisa, que levou à elaboração do conceito e do delineamento experimental da resposta de observação (Wyckoff, 1952, 1969), teve origem nas questões colocadas ao longo desse debate.

\section{Sobre os Autores}

P.E.

orcid.org/0000-0001-8652-2405

Universidade de São Paulo

(USP) - São Paulo, SP

peterendemann@hotmail.com

\section{Direitos Autorais}

Este é um artigo aberto e pode ser reproduzido livremente, distribuído, transmitido ou modificado, por qualquer pessoa desde que usado sem fins comerciais. 0 trabalho é disponibilizado sob a licença Creative Commons CCBY-NC 
As questões que foram fundamentais para o problema de pesquisa de Wyckoff $(1952,1969)$ e serão tratadas na apresentação desta pesquisa são: (a) definição da discriminação e da seletividade em autores como Skinner (1933, 1934, 1938), Spence $(1936,1940,1945)$, Lashley (1929, 1942) e Krechevsky (1932, 1938); (b) o controle da resposta de orientação dos receptores e do contato sensorial do organismo com os estímulos; e (c) variáveis do treino discriminativo, como as medidas, os diferentes momentos do treino a partir do qual as medidas foram obtidas e, sobretudo, a manipulação do arranjo de estímulos.

Sobre as variáveis do treino, as medidas foram prioritariamente o número de acertos (respostas em condição de reforço) e erros (respostas em condição de extinção) ao longo do treino discriminativo. Essas medidas eram obtidas e calculadas em momentos distintos do treino. 0 momento do treino mais importante para se compreender o debate a ser apresentado foi nomeado como período pressolução, em que as respostas dos sujeitos experimentais ainda não haviam se diferenciado nas condições (reforço e extinção) programadas para o treino discriminativo.

Os estímulos foram arranjados de diferentes maneiras: desde arranjos mais simples aos mais complexos. Talvez seja essa uma das variáveis mais importante para se compreender os desdobramentos que levaram Wyckoff a elaborar seu problema de pesquisa. Os termos utilizados para referência a aspectos funcionais dos estímulos foram diversos ao longo da literatura revisada. Os estímulos discriminativos S+ e S- são referidos como discriminativos, relevantes ou correlacionados ao reforço. Além desses estímulos, uma classe particular destes foi discutida e utilizada nos procedimentos: os estímulos irrelevantes (Si) ou não correlacionados. Estes últimos são aqueles que não são correlacionados ao reforço ou à sua ausência, mas que podem estar presentes na situação experimental e, mais especificamente, estar presentes no momento da produção do estímulo reforçador. Por exemplo, partes da caixa experimental, posição na qual os estímulos discriminativos são apresentados etc.

O controle de respostas sensoriais era planejado pela manipulação do arranjo de estímulos com o objetivo de se investigar exclusivamente o processo de estabelecimento de respostas diferenciais na presença de estímulos fisicamente distintos (Spence, 1940). Como será visto, o controle da resposta sensorial e do contato do sujeito experimental com os estímulos determinou as diferentes definições de discriminação postas em debate.

O exame histórico dos estudos sobre o estabelecimento da discriminação das décadas de 1930 e 1940, e das questões que determinaram o problema de pesquisa de Wyckoff $(1952,1969)$, teve como objetivo resgatar a definição do processo de discriminação operante e as contribuições de
Wyckoff no que se refere ao papel das respostas sensoriais para a discriminação.

A presente pesquisa, por sua vez, foi conduzida com base nas referências dos artigos de Wyckoff. A leitura destes, e dos artigos por ele referidos, conduziu à análise das principais questões teóricas e experimentais que permearam as discussões sobre o estabelecimento da discriminação nas décadas de 1930 e 1940. A apresentação desta pesquisa foi organizada da seguinte maneira: primeiramente, serão apresentados o conceito e o delineamento de resposta de observação; em seguida, os estudos sobre o estabelecimento da discriminação nas décadas de 1930 e 1940, e os aspectos relevantes da Controvérsia Continuidade vs. Descontinuidade para a elaboração do problema de pesquisa de Wyckoff (1952, 1969); e, por fim, serão retomados o papel da resposta de observação para o estabelecimento da discriminação e algumas considerações finais.

\section{A RESPOSTA DE OBSERVAÇÃO}

De modo geral, a resposta de observação é aquela que coloca o organismo em contato sensorial com os estímulos. Em contingências de discriminação, a resposta de observação produz os estímulos sob controle, dos quais outra resposta será diferenciada em função do treino de reforçamento diferencial. Nesse sentido, Wyckoff (1952) estabeleceu uma importante diferença funcional entre duas classes operantes de respostas, ambas relevantes para o estabelecimento da discriminação: respostas cuja função é a produção de consequências diferenciais, nomeadas por ele como respostas efetivas, e as respostas cuja única função é a exposição do organismo aos estímulos discriminativos, as respostas de observação.

Frente às dificuldades tecnológicas da época, Wyckoff (1969) elaborou um procedimento, conhecido como delineamento de observação, que contou com dois diferentes manipulandos adaptados a uma caixa de condicionamento operante. As respostas a cada manipulando exerciam funções distintas e independentes para o estabelecimento de uma discriminação sucessiva: (a) um disco de resposta alocado na parede da caixa, servindo como manipulando principal para as respostas efetivas, cujo bicar no disco de resposta era seguido de reforço a depender do componente em vigor; (b) um pedal alocado no piso da caixa, servindo como manipulando para as respostas de observação, cujo pisar no pedal fazia com que os estímulos discriminativos fossem apresentados na chave de resposta.

Ao todo, 20 pombos assumiram função de sujeitos experimentais. Os estímulos utilizados foram as luzes vermelha (S+), verde (S-) e branca. A luz branca serviu para iluminar a chave de resposta quando o pedal não estava 
sendo pressionado e, nesse sentido, era irrelevante para a discriminação, não sinalizando nenhum dos componentes programados.

Após uma fase de adaptação às condições experimentais, os sujeitos foram submetidos a um treino de reforçamento diferencial. Componentes positivos (com reforço - acesso ao comedouro por 4s) e negativo (sem reforço) foram intercalados sucessivamente. Os reforços a respostas de bicar na chave de respostas foram programados em um esquema $\mathrm{FI}$ 30s. Durante o treino, a luz vermelha acompanhava os componentes de reforço e a luz verde acompanhava os componentes de extinção. As luzes eram produzidas na chave de resposta somente enquanto o sujeito pisasse no pedal, isto é, emitisse a resposta de observação. Caso contrário, o disco permaneceria iluminado de branco e as respostas de bicar o disco produziriam o reforço, a depender de qual esquema estivesse em vigor. Em resumo, pisar o pedal, emitindo a resposta de observação, transformava um esquema misto (não sinalizado) em um esquema múltiplo (sinalizado) de mesmo valor.

Depois das seis primeiras sessões da Fase Experimental, os sujeitos foram distribuídos em três grupos (A, B e C). 0 grupo A permaneceu no treino discriminativo (reforçamento diferencial) e, assim, a resposta de observação continuava a produzir as luzes verde e vermelha e estas, por sua vez, mantinham-se correlacionadas com o extinção e reforço, respectivamente. Para o grupo $\mathrm{B}$, a relação dos estímulos com o reforço foi revertida (o estímulo relacionado ao $\mathrm{FI} 30$ s passou a ser o verde, e o estímulo relacionado à extinção passou a ser o vermelho); e o grupo C foi colocado em condição de reforçamento não-diferencial, na qual as luzes, apesar de produzidas pela pressão no pedal, não estavam mais correlacionadas ao reforço. Este poderia ser produzido diante de verde, vermelho (se o pedal estivesse pressionado) ou branco (quando o pedal não estivesse pressionado).

Wyckoff (1969) registrou o tempo em que o pedal era pressionado, isto é, a duração das respostas de observação. Com isso, ele analisou variações na duração da resposta de observação em função da produção ou não dos estímulos correlacionados com o reforço. A duração da resposta de observação na primeira fase (treino de reforçamento diferencial) começou alta para todos os sujeitos, e assim permaneceu para o grupo $A$, que se manteve no treino discriminativo. Para o grupo $B$, a duração da resposta de observação diminuiu logo que a correlação dos estímulos foi revertida, mas aumentou assim que a discriminação foi novamente estabelecida. Para o grupo $C$, que foi submetido a reforçamento não-diferencial (as luzes podiam ser produzidas, mas não havia correlação), a duração da resposta de observação diminuiu, assim permanecendo.

Com base nos resultados, Wyckoff (1969) argumenta que "sob condições de reforçamento diferencial, a probabilidade da resposta de observação $(P o)$ irá aumentar, e sob condições de reforçamento não-diferencial a probabilidade irá diminuir " (p. 238). Os padrões encontrados na duração da resposta de observação indicaram que as condições de condicionamento (reforçamento diferencial) e extinção (reforçamento não-diferencial) afetaram, de forma consistente, a resposta de observação. Em conclusão, Wyckoff (1952) afirma que "a exposição a estímulos discriminativos terá efeito reforçador sobre a resposta de observação à medida que o sujeito aprende a responder diferencialmente aos estímulos" (p. 435).

\section{A DISCRIMINAÇÃO NOS ANOS 1930 E 1940 E A CONTROVÉRSIA CONTINUIDADE VS. DESCONTINUIDADE}

Segundo Skinner (1938), a discriminação é o processo seletivo pelo qual o organismo vem a responder a uma parte do ambiente e não a outras. Para Skinner, a discriminação não se refere apenas a "perceber" que os estímulos no ambiente são diferentes, mas sobretudo a comportar-se diferencialmente sob controle dos estímulos. Esse processo é estabelecido pelo treino de reforçamento diferencial ou treino discriminativo, envolvendo o condicionamento e a extinção.

0 treino de reforçamento diferencial (uso da extinção) é necessário porque apenas o condicionamento de respostas na presença de determinados estímulos não garante que as respostas ocorrerão no futuro sob controle exclusivo daqueles estímulos, não garantindo, assim, o responder diferencial e a seletividade planejados pelo treino (Skinner, 1938). Caso haja apenas o condicionamento da resposta na presença de um estímulo, outros estímulos com propriedades físicas similares (indução/generalização) ou estímulos irrelevantes presentes no ambiente (condicionamento acidental) poderão controlar a resposta em ocasiões futuras.

Sobre as condições experimentais, Skinner (1938) argumenta que estas condições devem ser simplificadas de modo a minimizar o controle por estímulos irrelevantes (oriundos de histórias passadas) e a não permitir o condicionamento acidental de respostas a novos estímulos irrelevantes presentes no ambiente imediato. A simplificação pode ser feita pela remoção prévia dos estímulos irrelevantes (Si). Esta remoção poderia ser realizada, segundo Skinner (1938), "ao conduzir os experimentos em caixas à prova de som, escuras, com paredes lisas" (p. 55) etc. Nestes exemplos, os estímulos irrelevantes removidos seriam a presença de diferentes sons, luzes e texturas, bem como variações nesses estímulos. Entretanto, nem todo estímulo irrelevante pode ser removido. Mesmo em uma situação ótima de simplificação restariam, por exemplo, os "sons produzidos pelos movimentos do rato ou a estimulação tátil 
da caixa" (Skinner, 1938, p. 55), e ainda a localização espacial em que os estímulos S+ e S- são apresentados.

De acordo com Skinner (1938), a correlação do estímulo reforçador com o S+ faz com que o estímulo reforçador seja liberado a respostas na presença de $\mathrm{S}+\mathrm{Si}$. Segundo o autor, três relações podem ser estabelecidas a partir deste fato: S+.R, S+Si.R e Si.R. Este triplo condicionamento, como chamou Skinner, não pode ser completamente evitado e, uma vez que os estímulos irrelevantes podem gerar problemas, "eles são usualmente eliminados pela extinção. A discriminação é estabelecida quando respostas a S+Si são reforçadas e respostas a Si são extintas" (Skinner, 1938, p. 177). Desse modo, o condicionamento acidental é eliminado e a discriminação sucessivamente estabelecida. Nas palavras de Skinner (1938), “a discriminação é o acúmulo das pequenas diferenças que são em si mesmas propriedades do comportamento original do organismo" (p.170).

Spence (1936) parte de princípios semelhantes aos de Skinner (1938) para discutir a natureza do estabelecimento da discriminação. Segundo Spence, a discriminação é estabelecida a partir da aplicação sucessiva do condicionamento e da extinção, bem como da correlação entre os estímulos antecedentes e o estímulo reforçador. No entanto, Spence (1936) vai discutir o estabelecimento da discriminação por meio do fortalecimento e enfraquecimento de tendências excitatórias, adquiridas pelos estímulos ao longo do treino discriminativo. Segundo Spence (1936), "o aprendizado da discriminação envolve, sobretudo, o fortalecimento relativo de tendências excitatórias de certos componentes de um estímulo complexo quando comparado a outros componentes até o ponto em que isto alcance força suficiente para determinar diferencialmente a resposta" ( $p$. 439).

Segundo Spence (1936), em uma situação de discriminação haverá sempre vários estímulos, entre eles, os S+, S- (correlacionados) e Si (não correlacionados - irrelevantes). Estímulos irrelevantes, via condicionamentos passados, possuem tendências excitatórias diversas e, com isso, podem determinar as respostas no início do treino. Segundo o mesmo autor, tendências excitatórias responsáveis pela resposta a estímulos irrelevantes são controladas ao longo da treino discriminativo, simultâneo à produção de variações nas tendências excitatórias de S+ e S-.

De acordo com Spence (1936), os estímulos S+Si que incidam sobre o aparato sensorial dos organismos no momento crítico em que o estímulo reforçador é liberado adquirem tendência excitatória. Do mesmo modo, os estímulos S-Si, ao longo da extinção, adquirem tendências inibitória. Como o condicionamento é consistente apenas com o S+ e a extinção, com S-, o treino produzirá diferenças nas tendências excitatórias destes estímulos e os estímulos
Si manterão suas tendências equalizadas por estarem presentes em ambas as condições.

Em resumo, tanto para Skinner (1938) como para Spence (1936) havia a preocupação em simplificar o máximo possível a situação experimental, de modo a promover o contato com os estímulos S+ e S-. Skinner (1938) defende que, não sendo possível a remoção de $\mathrm{Si}$, deve-se diminuir sua intensidade e aumentar a intensidade de S+. Segundo Spence (1940), "os interesses estão centrados na investigação do processo de aprendizagem em situações em que a resposta perceptiva é tão simples quanto possível" (p. 278). Isso reflete seus comprometimentos em investigar o processo de estabelecimento de respostas diferenciais aos estímulos (S+ e S-), em função do treino de reforçamento diferencial em detrimento da análise de fatores sensoriais.

A discriminação é definida em Skinner e Spence como um processo cumulativo e contínuo. O processo contínuo ocorre porque todos os estímulos que incidam no aparato sensorial do organismo no decorrer no treino discriminativo são condicionados ou extintos (Skinner, 1938), ou adquirem ou perdem tendência excitatória (Spence, 1936). Visto que o Si está presente em ambas as condições, o controle por Si é gradualmente eliminado ou equalizado, e as respostas passam a ser determinadas apenas por S+ e S-. Esta definição do que seja o processo da discriminação ficou conhecida como a teoria da continuidade, tendo Spence como o grande expoente.

A posição contrária à da continuidade foi desenvolvida por Lashley $(1929,1938,1942)$ e Krechevsky (1932, 1937, 1938), e ficou conhecida como teoria da descontinuidade. 0 debate entre as posições contrárias ficou conhecido como a Controvérsia Continuidade vs. Descontinuidade. Segundo Lashley $(1929,1942)$ e Krechevsky (1932), a discriminação é estabelecida pelo treino discriminativo (condicionamento e extinção), tendo como produto um responder diferencial sob controle de S+ e S-. A questão principal que diferenciará as posições no debate é sobre a seletividade no processo de estabelecimento da discriminação. Segundo os autores da descontinuidade, não há necessariamente o condicionamento e a extinção gradual dos estímulos S+ e S-, nem tampouco uma eliminação ou equalização gradual do controle exercido por $\mathrm{Si}$, mesmo que esses estímulos incidam sobre o aparato sensorial do organismo. Admitir a discriminação como um processo cumulativo e contínuo seria ignorar aspectos relevantes dos mecanismos sensoriais, que afetam 0 estabelecimento das respostas diferenciais, especialmente a seletividade. Para melhor compreender essa diferença vejamos a análise de Lashley (1929).

Lashley (1929) analisou o desempenho de ratos em um aparato onde eles deveriam saltar em direção a uma de duas diferentes luzes (S+ e S-). As luzes foram apresentadas randomicamente em duas posições distintas. A análise foi 
realizada a partir do momento em que o animal foi colocado no aparato, ou seja, desde o início do treino discriminativo. Os sujeitos experimentais passaram um tempo saltando em apenas uma direção, independentemente da luz apresentada nela. Após um tempo, o sujeito passou a saltar em direções randômicas, em acordo com a apresentação das luzes. Passou a saltar, portanto, apenas em direção ao S+.

Por uma análise continuísta: no início do treino, tanto a localização como a luz incidiam sobre o aparato sensorial do sujeito. O sujeito saltava apenas a uma única direção, assim, a direção (Si) ora era seguida do reforço ora não, a depender da luz presente naquela direção. Ao mesmo tempo, o reforço só era liberado na presença de uma das luzes e nunca era liberado na presença da outra. Com o avanço do treino, o controle exercido pela direção deixa de ocorrer enquanto a luz passa a controlar a resposta, independentemente de sua localização.

Por uma análise não-continuísta: no início do treino, tanto a localização como a luz incidiam sobre o aparato sensorial do sujeito. O sujeito saltava apenas a uma única direção, assim, a direção (Si) ora era seguida do reforço ora não. Enquanto saltava nessa direção, as luzes não estavam necessariamente adquirindo gradualmente controle ou tendências excitatórias ou inibitórias. 0 animal respondia sob controle da direção e, dada a sua irrelevância, ele variava e respondia sob controle das luzes, passando a fazê-lo diferencialmente, em função das contingências programadas.

A sutil diferença entre as posições pode ser identificada no fato de que, durante as respostas sob controle da direção, o animal pode não estar aprendendo nada em relação aos estímulos (S+ e S-) programados, mesmo que estes incidam sobre o aparato sensorial. Dadas as opções e complexidade do arranjo de estímulos, o animal poderia variar entre as opções e, por muito tempo, não responder sob controle dos estímulos programados, para que as contingências fossem efetivas em estabelecer a discriminação. Para os nãocontinuístas, apesar de relações seletivas serem estabelecidas pelo treino, há mecanismos de seletividades que são anteriores ao treino e que podem afetar o estabelecimento da discriminação. Dessa forma, não seria possível definir o estabelecimento da discriminação como um processo contínuo.

Respostas a Si (como a direção), segundo Lashley, são "respostas à posição, à alternação, a dicas oriundas dos movimentos do pesquisador e que comumente precedem as respostas às luzes e representam tentativas de solução (...) são respostas que já faziam parte de uma gama de atividade do rato" (Lashley, 1929, p. 135) e são, assim, "trazidas" para a nova situação. Uma vez que essas respostas "fracassam" frente às contingências programadas, os sujeitos variam e passam a responder a outras propriedades do ambiente, que não necessariamente aos estímulos programados ( $\mathrm{S}+\mathrm{e} \mathrm{S}-$ ). $\mathrm{A}$ emissão dessas respostas (tentativas de solução) e a produção ao acaso do reforço em relação aos estímulos programados definem $\mathrm{o}$ que foi chamado de período pressolução.

Krechevsky (1932) analisou o desempenho de ratos albinos submetidos a um treino de discriminação entres brilhos em um labirinto tipo $\mathrm{T}$ ao longo do período pressolução. Krechevsky observou que respostas a diferentes Si's eram sistemáticas ao longo de intervalos de tempo. Krechevsky (1932) afirmou ainda que o animal, no começo do treino, não estaria em "uma situação confusa, conglomeração sem sentido de impressões sensoriais para a qual responde de modo não-coordenado" (p. 528), nem tampouco passaria a responder de modo coordenado, sistemático e seletivo em função de um efeito cumulativo das contingências diferenciais. Segundo o autor, controles seletivos estão ocorrendo de forma coordenada. Em algum momento do treino, o animal não apenas entra em contato sensorial com os estímulos ( $\mathrm{S}+$ e $\mathrm{S}-$ ), mas passa a responder sob controle desses estímulos. Nesse momento, apesar de passar a responder sob controle dos estímulos ( $\mathrm{S}+$ e $\mathrm{S}$-), suas respostas ainda são indiferenciadas em relação a eles. Contudo, as contingências diferenciais entram em vigor e o responder diferencial e uma nova relação seletiva entre o animal e seu ambiente são estabelecidos.

De acordo com o princípio da seletividade (Lashley, 1929), nem o animal responde a todos os estímulos que incidem sobre seu aparato sensorial nem, ao longo do condicionamento, todos esses adquirem tendências excitatórias. E isso interditaria resumir a discriminação a um processo contínuo, envolvendo o acúmulo de pequenas diferenças na frequência das respostas, aos estímulos correlacionados. Segundo Krechevsky (1932), o estabelecimento da discriminação envolveria séries de sistemáticas respostas a diferentes estímulos dispostos ao longo do tempo na situação experimental. Em Krechevsky (1932), esta série de respostas sistemáticas representa o que Lashley (1929) chamou de tentativas de solução. Tanto essa série de respostas como a suscetibilidade inicial a determinados estímulos e não a outros (seletividade) demonstrariam relativa independência às contingências diferenciais programadas. Em referência a Spence (1936), essa relativa independência poderia ser dita da seguinte maneira: nenhuma tendência excitatória ou inibitória estará sendo necessariamente adquirida pelos estímulos S+ e Scaso o animal esteja respondendo sob controle de Si.

Spence $(1936,1940)$, responderá às criticas apresentando o estudo de McCulloch e Pratt (1934), o que, segundo Spence, reforça seus argumentos em detrimento dos de Lashley (1929) e de Krechevsky (1932). O procedimento elaborado por McCulloch e Pratt consistia em reversões entre os estímulos S+ e S- (o que era S+ passava a ser S- e o vice 
-versa), ainda no período pressolução. Caso estes estímulos $\mathrm{S}+$ e S- estivessem adquirindo e/ou perdendo tendências excitatórias, isso seria evidenciado em atraso no estabelecimento da discriminação com animais que passaram pela reversão em comparação com outros que não passaram.

Esse procedimento envolvendo a reversão das funções discriminativas dos estímulos $\mathrm{S}+$ e $\mathrm{S}$-, ainda no período pressolução, foi o procedimento-chave ao longo de toda a controvérsia. De modo geral, se a reversão das funções discriminativas no período pressolução afetasse a extensão desse período (medido por grupo controle), isso indicaria que os estímulos estavam adquirindo tendências excitatórias ou inibitórias desde o início do treino. Caso não afetasse a extensão do período pressolução, indicaria que o animal estava respondendo sob controle de Si's e que nada estava sendo aprendido em relação aos estímulos S+e S-.

Segundo McCulloch e Pratt (1934), em um treino de discriminação definido como "difícil" (e.g., pequena diferença física entre os estímulos), os animais frequentemente permanecem ou passam a responder sistematicamente à posição. Se o responder sistemático ao Si (posição), "servisse para inibir a manifestação das respostas 'corretas', o acúmulo das diferenças não poderia ser tomado como representativo da discriminação" (McCulloch \& Pratt, 1934, p. 272). Por outro lado, se for demonstrado que mesmo nessas condições ocorre o acúmulo, tanto este poderia ser representativo da discriminação, como a soma dos escores poderia ser utilizada para previsão do momento em que a discriminação será estabelecida.

McCulloch e Pratt (1934) realizaram um treino de discriminação, com 120 ratos, envolvendo dois pesos diferentes, 25 e 75 gramas, colocados em bandejas e que deveriam ser empurradas por $110 \mathrm{~cm}$ sobre uma chapa de metal bifurcada. As bandejas contendo esses pesos estavam randomicamente dispostas no lado direito ou esquerdo. As respostas exigidas do animal foram empurrar a bandeja por $110 \mathrm{~cm}$ para ter acesso à comida, quando correta. Em uma mesma tentativa, o animal poderia empurrar a bandeja até $70 \mathrm{~cm}$ sem que isso configurasse um erro ou acerto e, assim, retornar e empurrar a outra bandeja disponível. Os animais foram divididos em 5 grupos e passaram por diferentes treinos. Os grupos foram formados para permitir várias análises, inclusive o efeito de supertreino (overtraining effect). Os dois grupos de interesse foram o grupo-controle e o grupo que passou por reversão (grupo II) na tentativa 24, ainda no período pressolução. Os resultados foram apresentados pelo número de erros. 0 grupo que passou pela reversão demonstrou um maior número de erros.

Com base nos resultados, McCulloch e Pratt (1934) concluíram que houve, sim, um efeito cumulativo do treino em relação aos estímulos relevantes, mesmo no período pressolução, no qual as respostas eram determinadas prioritariamente pela posição esquerda/direita. Segundo os autores, "Em face destes resultados, os argumentos de Lashley sobre a natureza e os efeitos da formação inicial, não podem ser considerados aplicáveis nas condições utilizadas neste experimento" (McCulloch \& Pratt, 1934, p. 286). Segundo os autores e reafirmado em Spence (1936), mesmo que o animal esteja emitindo respostas a Si e mesmo que isto possa ser configurado como uma tentativa de solução (e.g., respostas sistemáticas à posição), os estímulos relevantes são relacionados a um efeito cumulativo.

Com o objetivo de testar se o número de erros após a reversão era, de fato, proporcional ao número de tentativas de treino, Krechevsky (1938) realizou um outro experimento em discriminação visual. Os sujeitos foram 47 ratos, colocados em um aparato de salto. Os estímulos utilizados foram cartas brancas com linhas pretas horizontais e verticais. Os animais foram divididos em três grupos. 0 procedimento utilizado consistia na reversão dos estímulos S+ e S-, ainda no período pressolução, em diferentes tentativas. 0 grupo I foi o grupocontrole sem reversão, e os grupos II e III passaram por reversões nas tentativas 20 e 40, respectivamente. Os dados obtido foram de acordo com todos os argumentos já apresentados aqui, por Krechevsky, ou seja, a reversão não afetou a extensão do período pressolução. Com base nos resultados, Krechevsky (1938) acrescenta que os efeitos do condicionamento e extinção atuarão para o estabelecimento da discriminação apenas quando o animal passar a atentar seletivamente para os estímulos relevantes ( $\mathrm{S}+$ e $\mathrm{S}$-), ou apenas quando esses estímulos estiverem associados a uma tentativa de solução específica.

Spence (1940), ao analisar a situação experimental dos estudos, insere uma questão importante em seus argumentos. O autor afirma que a mera presença dos estímulos na situação experimental não garante que estes incidam no aparato sensorial do animal. Segundo Spence (1940), o papel da seletividade descrito por Krechevsky (1937) é de fato uma questão importante, mas, para a análise do estabelecimento da discriminação, é extremamente importante que os estímulos relevantes ( $\mathrm{S}+$ e $\mathrm{S}$-) incidam sobre o aparato sensorial desde o início do treino. Ainda de acordo com Spence (1940), "Caso a situação de discriminação seja de um tipo que o aparato sensorial do animal não seja forçado a receber os estímulos relevantes desde o início do treino, as associações não serão formadas entre as respostas e estes estímulos" (p. 276).

Spence $(1940,1945)$ defende que, em situações nas quais os estímulos especificados não incidem sobre o aparato sensorial, "o animal é requerido a aprender, em adição à resposta seletiva, uma resposta perceptual apropriada que o conduza para a recepção dos estímulos relevantes (...) o animal deve aprender a orientar e fixar a cabeça e os olhos a 
fim de receber os estímulos críticos" (Spence, 1940, p. 276). Segundo Spence (1940) também, estas respostas serão aprendidas pelas mesmas razões que são aprendidas as respostas de empurrar as bandejas, ou saltar em direção a um estímulo, ou passar pela portinhola que contenha o estímulo reforçador, etc.

A necessidade de se aprender outra resposta no momento em que os estímulos correlacionados não incidem (por estarem em lugares diversos da situação experimental) no aparato sensorial do organismo, foi, em parte, analisada experimentalmente em Ehrenfreund (1948) com ratos. Ehrenfreund realizou dois experimentos de discriminação com reversão no período pressolução, utilizando um aparato semelhante ao de Krechevsky (1938). Os estímulos utilizados foram cartas brancas com triângulos pretos. Em ambos os experimentos, os estímulos foram apresentados de modo balanceado em relação à posição esquerda/direita. A manipulação que diferenciou os experimentos foi apenas a posição dos estímulos no interior das cartas. A posição dos estímulos especificados em relação ao operando (a passagem pela qual o rato tinha acesso à comida) foi manipulada. Manipulação esta que, segundo os autores, permitiria apontar para uma solução ao debate.

No experimento 1, os estímulos especificados foram apresentados na parte superior das cartas, estabelecendo uma distância em relação ao operando (ou à parte superior da passagem) de $2 \mathrm{~cm}$. Os animais foram divididos em dois grupos. 0 grupo-controle não passou por reversão, e o grupo experimental, sim. A reversão para este grupo ocorreu na tentativa 40. Os resultados foram de acordo com o que é previsto pela teoria da descontinuidade. A partir do mesmo procedimento utilizado no experimento 1 , no experimento 2 , os estímulos especificados foram apresentados na parte inferior das cartas, localização específica onde, segundo Spence $(1940,1945)$, os ratos fixavam a cabeça e olhos antes do salto. Os resultados foram de acordo com a teoria da continuidade, inclusive sobre aquisição de tendências excitatórias diferenciais por parte de S+ e S-, mesmo quando se observou respostas sistemáticas a Si (no caso posição). Resultados semelhantes foram obtidos em Bitterman e Coate (1950) e Jeeves e North (1956).

Uma questão, que aos poucos foi ganhando espaço no debate, foi experimentalmente manipulada no estudo de Ehrenfreund (1948): o arranjo no qual os estímulos são dispostos. Com base nos dados, Ehrenfreund reafirmou o que fora defendido por Spence (1940), ao lembrar que respostas a Si acompanham a aquisição de tendências excitatórias diferenciais por S+ e S-, quando estes são arranjados de tal modo que incidam sobre o aparato sensorial do animal. No contrário, os estímulos S+ e S- não adquirem tais diferenças. Para que estes estímulos adquiram-nas, será preciso que o animal aprenda outra resposta: a resposta de orientação apropriada dos receptores (Spence, 1940).

Em conclusão, ao inserir a necessidade de uma resposta de orientação dos receptores no debate, Spence (1940) admite que o animal pode estar respondendo ao Si e não estar aprendendo nada em relação aos estímulos relevantes. Mas, nesse caso, segundo Spence, não estaria ocorrendo o estabelecimento da discriminação, insistindo no controle da resposta sensorial para a investigação dos processos envolvidos no estabelecimento das respostas diferenciais.

\section{O PAPEL DA RESPOSTA DE OBSERVAÇÃO PARA 0 ESTABELECIMENTO DA DISCRIMINAÇÃO}

Segundo Wyckoff (1952), Spence (1936, 1940) desenvolveu "uma teoria sobre o estabelecimento da discriminação a partir de situações em que nenhuma resposta de observação foi requerida, a partir de situações onde, segundo Spence, a exposição aos estímulos discriminativos era certa de ocorrer" (Wyckoff, 1952, p. 431). A inserção da resposta de observação nos estudos sobre a discriminação foi estabelecida a partir de condições nas quais o aprendizado de uma resposta de observação apropriada foi requerida. Esse requerimento foi indicado por Wyckoff (1952) pela probabilidade de ocorrência da resposta de observação $(P o)$ no início do treino discriminativo, $0<P o$ $<1$, onde $P o=1$ significaria uma situação, como elaborada por Spence, na qual nenhuma resposta de observação é requerida pela simplificação da situação experimental. Em tais condições, a resposta de observação operante, cuja função é colocar o organismo em contato com os estímulos discriminativos, não é requerida, e o contato é garantido por fatores respondentes ou endógenos (Connor, Egeth, \& Yantis, 2004; Yantis, 2000).

Em uma revisão sobre discriminação espacial com macacos, Stollnitz (1965) levanta questões relacionadas à probabilidade da resposta de observação $(P o)$. Segundo o autor, se o animal não for exposto aos estímulos discriminativos, não haverá responder diferencial em relação a eles. Com efeito, toda tarefa de discriminação requer uma resposta de observação. Entretanto, e de forma extremamente interessante, o autor coloca que "paradoxalmente, as situações nas quais a resposta de observação é mais provável de ocorrer $[P o=1]$ são justamente aquelas nas quais sua necessidade é ignorada" (Stollnitz, 1965, p. 249). Stollnitz reformula a frase de Wyckoff (1952) sobre o requerimento das respostas de observação em situações tais quais elaboradas por Spence (1936, 1940). Segundo Stollnitz (1965), o que ocorre é que nessas situações não há o requerimento de que uma nova resposta de observação seja adquirida, pois a resposta de observação 
apropriada já está estabelecida ou é favorecida por aspectos do arranjo de estímulos.

Segundo Wyckoff (1952), a simplificação da tarefa, de modo a minimizar o aprendizado das respostas de orientação, poderia impor limites no valor preditivo das teorias sobre o estabelecimento da discriminação, pois nem mesmo em situações controladas de laboratório seria possível precisar se os animais serão expostos aos estímulos. Como afirmou Wyckoff (1952), Ehrenfreund (1948) demonstrou que o arranjo de estímulos pode favorecer ou não o contato sensorial do organismo com tais estímulos e, desse modo, vir a requerer a aquisição de respostas de orientação dos receptores e, com isso, afetar a extensão do período pressolução.

\section{CONSIDERAÇÕES FINAIS}

No contexto do debate anteriormente apresentado, Wyckoff (1952) discutiu o papel de respostas tais como "de atentar, de orientação, de perceber, atividades ligadas à organização sensorial, etc." (p. 431) no estabelecimento da discriminação, introduzindo o termo resposta de observação. Apesar de a função das respostas de orientação e, por consequência, das respostas sensoriais em contingências de discriminação ter sido definida por Spence (1940), foi Wyckoff (1969) quem originalmente a investigou em condições experimentais e operantes.

Com base na definição dessa função, Wyckoff (1969) delineou seu experimento colocando a produção dos estímulos discriminativos contingente à emissão de uma resposta distinta daquela que produz as consequências diferenciais programadas pelo treino discriminativo. Wyckoff (1969) isola experimentalmente "uma resposta que é prontamente mensurável e arranja a situação experimental de modo que essa resposta [pisar no pedal] é alocada na função de resposta de observação" (p. 238, colchetes e itálico acrescentados). Wyckoff (1969) argumenta que não há impeditivos para se acreditar que os processos envolvidos na aquisição e fortalecimento da resposta de pisar o pedal sejam distintos dos processos envolvidos na aquisição e fortalecimento da resposta de orientação dos receptores. Com efeito, o autor elabora uma unidade funcional interdependente e proporciona um tratamento empírico e operante ao que se estava discutindo teoricamente sobre o papel das respostas sensoriais no estabelecimento da discriminação.

Problemas identificados no estabelecimento da discriminação podem ser devidos às falhas na aquisição das respostas de observação, apropriadas aos estímulos da contingência programada. Segundo Carlin, Soraci, e Strawbridge (2002), um importante desafio em contingênciaseducacionais está em desenvolver tecnologias comportamentais que sejam eficazes em estabelecer e manter o contato com os estímulos relevantes (atentar) e inibir o contato com aqueles que são irrelevantes (ignorar) nas contingências programadas. Com o avanço das tecnologias, especialmente de rastreamento dos movimentos oculares, pesquisas sobre controle de estímulos têm sido recentemente desenvolvidas, e ampliado o conhecimento sobre as variáveis envolvidas no controle das respostas relevantes (de observação e efetivas) para o estabelecimento da discriminação (Carlin, et al., 2002; Dube \& Mcllvane, 1999; Dube, Lombard, Farren, Flusser, Balsamo, \& Fowler, 1999; Dube, Lombard, Farren, Flusser, Balsamo, Fowler, \& Tomanari, 2003; Dube, Balsamo, Fowler, Dickson, Lombard, \& Tomanari, 2006; Perez, Endemann, Pêssoa, \& Tomanari, 2015).

\section{FINANCIAMENTO}

FAPESP - Fundação de Amparo à Pesquisa do Estado de São Paulo (\# 2014/13483-2)

CNPq - Conselho Nacional de Desenvolvimento Científico e Tecnológico (\#140636/2009-9)

\section{REFERÊNCIAS}

Bitterman, M. E., \& Coate, W. B. (1950). Some new experiments on the nature of discrimination learning in animals. Journal of Comparative \& Physiological Psychology, 43, 198-210. http://dx.doi.org/10.1037/h0063652

Carlin, M. T., Soraci, S. A., \& Strawbridge, C. (2002). Enhancing performances of individuals with mental retardation: Manipulations of stimulus structure. In S. A. Soraci (Ed.), Perspectives on fundamental processes in intellectual functioning: Visual information processing. Greenwich, CT: Able.

Connor, C. E., Egeth, H. E., \& Yantis, S. (2004). Visual attention: Bottom-up vs. top-down. Current Biology, 14, 850-852. https://doi.org/10.1016/j.cub.2004.09.041

Dube, W.V., \& Mcllvane, W.J. (1999). Reduction of stimulus overselectivity with nonverbal differential observing responses. Journal of Applied Behavior Analysis, 32 (1), 2533. http://dx.doi. org/10.1901/jaba.1999.32-25.

Dube, W. V., Balsamo, L. M., Fowler, T. R., Dickson, C. A., Lombard, K. M., \& Tomanari, G. Y. (2006). Observing behavior topography in delayed matching to multiple samples. The Psychological Record, 56, 233-244. https://doi.org/10.1007/BF03395547 
Dube, W. V., Lombard, K. M., Farren, K. M., Flusser, D. S., Balsamo, L. M., \& Fowler, T. R. (1999). Eye tracking assessment of stimulus overselectivity in individuals with mental retardation. Experimental Analysis of Human Behavior bulletin, 17, 8-14.

Dube, W. V.; Lombard, K. M.; Farren, K. M.; Flusser, D. S.; Balsamo, L. M., Fowler, T. R., \& Tomanari, G. Y. (2003). Stimulus overselectivity and observing behavior in individuals with mental retardation. In Soraci \& Soraci (Eds.), Visual information processing (pp. 109-123). London: Proeger.

Ehrenfreund, D. (1948). An experimental test of the continuity theory of discrimination with pattern vision. Journal of Comparative and Physiological Psychology, 41, 408-422. http://dx.doi.org/10.1037/h0060666

Jeeves, H. A., \& North, A. J. (1956). Irrelevant or partially correlated stimuli in discrimination learning. Journal of Experimental Psychology, 2, 90-99. http://dx.doi.org/10.1037/h0040570

Krechevsky, I. (1932). Hypotheses in rats. Psychological Review, 39, 516-532. http://dx.doi.org/10.1037/h0073500

Krechevsky, I. (1937). A note concerning "the nature of discrimination learning in animals". Psychological Review, 44, 97-104. http://dx.doi.org/10.1037/h0061460

Krechevsky, I. (1938). A study of the continuity of the problemsolving process. Psychological Review, 45, 107-133. http://dx.doi.org/10.1037/h0056641

Lashley, K. S. (1929). Brain mechanisms and intelligence. Chicago: University of Chicago Press, 1929.

Lashley, K. S. (1938). The mechanism of vision. XV. Preliminary studies of the rat's capacity for detail vision. Journal of General Psychology, 18, 123-193. http://dx.doi.org/10.1080/00221309.1938.9709894

Lashley, K. S. (1942). An examination of the continuity theory as applied to discrimination learning. Journal of Genetic Psychology, 26, http://dx.doi.org/10.1037/h0060666

McCulloch, T. L., \& Pratt, J. G. (1934). A study of the presolution period in weight discrimination by white rats. Journal of Comparative Psychology, 18, 271-290. http://dx.doi.org/10.1037/h0075422
Perez, W., Endemann, P., Pessóa, C. V. B. B., \& Tomanari, G. Y. (2015). Assessing stimulus control in a discrimination task with compound stimuli: Evaluating testing procedures and tracking eye fixations. The Psychological Record, 65, 83-88. https://doi.org/10.1007/s40732-014-0092-1

Skinner, B. F. (1933). The rate of establishment of a discrimination. Journal of General Psychology, 9, 302-350. http://dx.doi.org/10.1080/00221309.1933.9920939

Skinner, B. F. (1934). A discrimination without previous conditioning. Proceedings of the National Academy of Sciences, 20, 532-536.

Skinner, B. F. (1938). The behavior of organisms: an experimental analysis. New York: Appleton-Century-Crofts.

Spence, K. W. (1936). The nature of discrimination learning in animals. Psychological Review, 43, 437-449. http://dx.doi.org/10.1037/h0056975

Spence, K. W. (1940). Continuous versus non-continuous interpretations of discrimination learning. Psychological Review, 47, 271-288. http://dx.doi.org/10.1037/h0054336

Spence, K. W. (1945). An experimental test of the continuity and noncontinuity theories of discrimination learning. Journal of Experimental Psychology, 35, 253-266. http://dx.doi.org/10.1037/h0060794

Stollnitz, F. (1965). Spatial variables, observing responses, and discriminations learning sets. Psychological Review, 72, 247-261. http://dx.doi.org/10.1037/h0022005

Wyckoff, L. B. (1952). The role of observing responses in discrimination learning: Part I._Psychological Review, 59, 431-442. DOI: 10.1037/h0053932

Wyckoff, L. B. (1969). The role of observing responses in discrimination learning: Part II. In D. P. Hendry (Org.), Conditioned Reinforcement (pp. 237-259). Homewood, Illinois: The Dorsey Press.

Yantis, S. (2000). Visual perception: Essential readings. Philadelphia: Psychology Press.

Recebido em: 23/10/2017 Primeira decisão editorial em: 29/11/2017 Aceito em: $14 / 03 / 2018$ 